\title{
OSTRACODEN BIOCOENOSEN UND THANATOCOENOSEN IM EMS-ESTUARIUM (N.O. NIEDERLANDE)
}

\author{
C. W. WAGNER *)
}

(mit 7 Verbreitungstabellen)

\section{EINLEITUNG}

Im ökologischen Teil unserer Abhandlung über Holozän-Ostracoden der Niederlande ist eine Úbersicht von den uns bis jetzt bekannten Tatsachen, die die Ókologie von Ostracoden des Nordseegebietes betreffen, gegeben (Schrifttum 4, T. III).

$\mathrm{Da}$ auf diesem Gebiet noch sehr wenig Arbeit geleistet wurde, ist diese Übersicht recht fragmentarisch. So mangelt es uns an Daten über die Zusammenstellung der Ostracodenbiocoenosen im polyhalinen Wasser, deren Kenntnis von großem Belang ist im Verband mit den faunistischen Veränderungen welche beim Übergang vom Brackwasser nach dem marinen auftreten. Ganz allgemein kann gesagt werden, daß eine Untersuchung von einer Serie Proben, die genommen wurden in einem Gebiet, wo nacheinanderfolgend süßwasser, brack- und rein marine Bereiche vorkommen, eine wesentliche Vervollständigung unserer heutigen Kenntnis über die Ökologie der Ostracoden Nordwesteuropas darstellen könnte.

Beim ökologisch-sedimentpetrologischen Untersuch des Ems-Estuarium wurden diese Bedingungen erfüllt und deshalb haben wir gerne den Vorschlag von Herrn Dr. van Voorthuysen die Ostracoden dieses Gebietes zu bearbeiten, auf uns genommen.

Leider war es uns nicht möglich, die Proben selbst nehmen zu können. Deshalb begnügten wir uns mit der Untersuchung von Proben, die von Herrn Dr. vaN VoorthuYSEN zu Foraminiferenuntersuchungen genommen wurden.

Die für gute ökologische Studien nötige Trennung von lebenden und toten Exemplaren erreicht man mit Hilfe eines Farbstoffes, die im Beitrag von Herrn Dr. vaN VOORTHUYSEN näher beschrieben wird. Selbst-

-) Bataafse Internationale Petroleum Maatschappij N.V. (Royal/Dutch Shell Group), Den Haag. verständlich gibt dieser Färbungsvorgang nur annähernd an, ob eine Art in dem bestimmten Milieu tatsächlich lebt. Nach dem Tode des Organismus bleiben jedoch während kürzerer oder längerer Zeit die Weichteile erhalten und man kann sich vorstellen, daß ein totes Tier nach einem für diesen Organismus fremden Biotop transportiert wurde und trotzdem auf Grund der ,roten Farbe“ für "lebend“" gehalten wird. Die Klassifikation der Arten nach ihrer Biotopenempfindlichkeit nach Remane (Schrifttum 2) lehrt uns übrigens, da $B$ auch lebende Tiere aus ihrem eigentlichen Biotop geraten können. Um die wirklichen Assoziationen und ihre charakteristischen Biotope kennen zu lernen, ist also eine sehr detaillierte Studie nötig, wobei u.a. auch erforderlich ist, daß die Probenentnahme nicht nur einmal, sondern oft und zu verschiedenen Zeiten des Jahres erfolgen soll.

Dieses letztere ist außerdem von großem Belang, da viele Arten der Ostracoden nur einige Monate im Jahr leben und man bei einer einmaligen Probenentnahme, wie das bei unserer Untersuchung der Fall war, Gefahr läuft, eine Anzahl für ein bestimmtes Biotop charakteristischer Arten zu eliminieren. Vielleicht kann dadurch auch das Dominieren von Jugendexemplaren der Gattung Leptocythere in unserem Gebiet erklärt werden. Wenn genaue Angaben hinsichtlich des periodischen Entwicklungscyklus der Leptocythere-arten zur Verfügung ständen, würde dies vielleicht mit Hilfe der Daten dieser Probenentnahme bestätigt werden können.

Man sieht also, daß an unserer Untersuchung bereits einige Fundamentalfehler haften, welche jedoch, gesehen am Umfang dieser Studien und den uns zur Verfügung stehenden beschränkten Mitteln nicht vermieden werden konnten.

Demgegenüber steht jedoch die große Vielzahl von 
Gegebenheiten, welche gerade durch dieses Teamwork zur Verfügung standen und welche man bei selbständigen Untersuchungen fast immer entbehren muß, wie z.B. Salzgehaltbestimmungen, Bodenlithologie, Temperatur, Hydrologie u.s.w. Das Ganze bot uns also die Möglichkeit, unsere Kenntnis der Ostracodenökologie wesentlich zu vergrößern.

Da wir erst viel später als die anderen Mitarbeiter mit diesen Untersuchungen beginnen konnten, war es nicht möglich, alle Proben zu bearbeiten. Wir haben deshalb eine Wahl in diesem Sinne getroffen, daß wohl von allen Lebensräumen Proben untersucht wurden, jedoch nicht die Gesamtzahl, die dort genommen wurde. Angesichts der großen Gleichheit der Faunen in diesem Gebiet, hat diese Handlungsweise das allgemeine faunistische Bild bestimmt nicht beeinflußt.

Wie oben erwähnt, sind sowohl lebende als auch tote Exemplare (leere Schalen) in diesen Untersuchungen mit einbegriffen. Es war nämlich nicht allein unser Ziel, genauere Gegebenheiten über die Okologie zu bekommen, sondern auch nachzugehen in wie weit die Thanatocoenosen die Biocoenosis noch representieren. Wir haben in unserer Abhandlung über die Holozän-Ostracoden (Schrifttum 4) schon auseinandergesetzt, daß für eine Untersuchung der fossilen Holozän-Ostracodenfaunen (Paläothanatocoenosen), es notwendig ist, den ökologischen Wert der Thanatocoenosis zu kennen. Unsere Untersuchung setzt sich also sowohl aus dem Studium der Ostracoden-biocoenosen als auch aus der Untersuchung der Ostracoden-thanatocoenosen im Ems-Estuarium zusammen, das in sich selbst aus drei Gebieten besteht: die eigentliche Ems, das Dollartgebiet und das Gebiet zwischen Dollart und Rottumeroog (s. Ubersichtskarte).

Von den Rückständen der Proben wurden immer zwei Fraktionen untersucht: eine grobe Fraktion $(0.3-2 \mathrm{~mm})$ und eine mittlere $(0.15-0.3 \mathrm{~mm})$. Von jeder Probe wurden die grobe ganz und von der mittleren, falls vorhanden, 3 ,examining trays" untersucht.
Hinsichtlich der Terminologie der Lebensräume sind wir Remane (Schrifttum 2) gefolgt, der sie folgendermaßen einteilt:

I. Supralitoral (über der HW-Linie)

II. Eulitoral (Bereich zwischen normal HW und NW)

III. Sublitoral (Bereich unter der NW-Linie).

Die Einteilung des Brackwasserbereiches auf Grund des Verhaltens der Organismen übernahmen wir, wie auch schon früher (Schrifttum 4), von VäLIKangas (Schrifttum 3):

(0.2) $\quad 0.5 \% \mathrm{~S}=$ Süßwasser

$0.2 \quad(0.5)-2 \quad$ (3) $\% \mathrm{~S}=$ oligohalines Wasser

2 (3) -8 (10) $\% \mathrm{~S}=$ meio mesohalines Wasser

8 (10) - $16.5 \% \mathrm{~S}=$ pleio mesohalines Wasser

$16.5-\quad 30 \% \mathrm{~S}=$ polyhalines Wasser

$30 \% \mathrm{~S}=$ marines Wasser

$\mathrm{S}=$ Gesamtsalzgehalt.

Die Klassifikation der Bodenlithologie wurde von Wigcers zusammengestellt, u.zw. in seinen Beitrag dieses Symposiums.

An den Studien über lebende Ostracoden des EmsEstuarium hat auch die Biologin Frau A. P. C. DE Vos mitgearbeitet. Einige Monate vor Abschluß der Arbeit verschied sie im 65. Lebensjahr stehend. Wir wollen Ihrer gedenken, die ein Pionier in den Untersuchungen der rezenten Süß- und Brackwasserostracoden der Niederlande war. Durch die freundliche Vermittlung von Herrn Dr. STocx (Zoologisches Museum der Stadt Amsterdam) kamen wir in den Besitz ihrer Forschungsergebnisse über Ostracoden des EmsEstuarium, die wir in unseren eigenen Untersuchungen mitverarbeitet haben. Unsere Hoffnung besteht darin, $\mathrm{daB}$ die jetzigen Ergebnisse so sind, wie sie sich das vorgestellt hat.

Schließlich schulden wir noch Herrn Dr. O. Ezofson Dank, der uns Vergleichsmaterial zukommen lieB und auch Herrn Dr. G. J. A. Pokonny der die Ubersetzung dieser Abhandlung ins Deutsche lieferte.

\section{ALLGEMEINE BEMERKUNGEN UBER DIE OSTRACODENFAUNEN} DES EMS-ESTUARIUM

\section{a. Biocoenosen}

Es zeigte sich, daß die lebende Ostracodenfauna, die in den untersuchten Proben des Gebietes angetroffen wurde, sehr artenarm ist.

Nur die folgenden Arten wurden angetroffen:

Cyprideis torosa

Leptocythere castanea

\section{Leptocythere lacertosa *) \\ Leptocythere spp. juv. \\ Semicytherura sella \\ Elofsonia pusilla}

") Diese Art fassen wir sensu lato auf (Schrifttum 1a, S. 110). 
Paradoxostoma normani (sehr selten)

Cytherois fischeri (sehr selten).

Wie bereits in der Einleitung bemerkt, dominieren die juvenilen Formen der Gattung Leptocythere, das wohl im Zusammenhang stehen kann mit der nur einmaligen Probenentnahme aus diesem Gebiet und dem periodischen Entwicklungszyklus der Leptocytherearten. Die Jugendformen der verschiedenen Arten gleichen einander so sehr, daß eine Artbestimmung nicht zu verwirklichen ist. Aber auch die Artbestimmungen adulter Leptocytheren auf Grund ihrer Schalenmerkmale ist nicht leicht. Zwar gibt es eine von den Biologen aufgestellte Artendifferenziation auf Grund der Weichteile, aber es ist zunächst die Frage, ob alle von ihnen gebrauchten Artnamen nomenklatorisch zurecht bestehen. So wird von den Biologen u.a. der Artname Leptocythere pellucida (BAIrd, 1850) gebraucht. BaIRD hat aber 1850 nur die Schale dieser Art beschrieben und dazu noch unvollständig. Wir fragen uns daher, ob die von den Biologen auf Grund der Strukture der Weichteile zu Leptocythere pellucida gerechneten Exemplare wohl identisch sind mit der von BaIRD beschriebenen Art. Leider ist das Originalmaterial von BAIRD nicht mehr zugänglich, sodaß nicht einmal durch ein nochmaliges Studium von Schalenmerkmalen die Art genau bestimmt werden kann.

Eine weitere Schwierigkeit ist die, daß mehrere Leptocythere-arten von Biologen auf Grund der Weichteile aufgestellt wurden, ohne die Schalenmerkmale zu erwähnen. Um eine solche Art an Schalenmerkmalen bestimmen zu können, muß man sich an die Biologen wenden, damit man so auch das Schalenmaterial bekommt, wovon die Schalenmerkmale beschrieben werden können.

Selbstverständlich ist eine solche Artenbestimmung nach. Schalen sehr subjektiv, weil sie abhängig ist von der genauen Bestimmung des betreffenden Biologen. Der Wunsch, auch rezente Arten nach Schalenmerkmalen allein bestimmen zu können, wird deutlich, wenn man u.a. bedenkt, daß der Quartärpaläontologe nur leere Schalen findet von Arten, die auch noch rezent vorkommen, und er so fossiles und rezentes Material vergleichen können muß. Diese Fragenbereiche haben wir in unserer Abhandlung (Schrifttum 4, S. 11) näher beleuchtet.

Die Bestimmungen der Leptocythere-arten des Ems-Estuarium sind basiert auf Vergleichsmaterial der beiden Biologen Frau A. P. C. DE Vos und Herrn Dr. O. ELofson.

Unsere juvenilen Leptocythere-exemplare representieren wahrscheinlich mehr Arten, als die oben er- wähnten, aber wie schon früher gesagt, kann eine genaue Artenbestimmung nicht erlangt werden.

Cyprideis torosa wird in der biologischen Literatur im allgemeinen Cyprideis litoralis genannt. Wir haben jedoch bereits in unserer Publikation über die Holozän-Ostracoden auseinandergesetzt, daB der Name "torosa" Priorität hat (Schrifttum 4, S. 39, 40). Leider sind in dieser Publikation in der Beschreibung der neuen Gattung Elofsonia einige störende Fehler. Zunächst wurden auf S. 71 „Valve droite“ und „Valve gauche" verwechselt. Weiter machte man uns aufmerksam, daß die Struktur des Schlosses, so wie wir sie beschrieben, nicht ganz richtig ist. Das Schloß scheint (linke Klappe) noch eine kleine Zahngrube zu besitzen vor dem gekerbten Zahn, welcher tatsächlich den vordersten Teil des differenzierten Mittelschlosses representiert. Eine solche Schloßstruktur scheint auch besser übercinzustimmen mit dem allgemeinen Bild der Ostracodenschlösser. Schließlich sei auch noch erwähnt, daß die von uns beschriebene Erpetocypris chevreuxi-Art nicht chevreuxi, sondern $E$. reptans ist (Schrifttum 4, S. 29).

\section{b. Thanatocoenosen}

In den Proben unseres Gebietes haben wir eine große Anzahl leeren Schalen von Ostracoden gefunden, welche z.T. zu den oben erwähnten Arten gehören, doch der größere Teil der Schalen gehört zu Arten, welche lebend nicht im Ems-Estuarium vorkommen.

Diese Arten sind:

\section{Süß-bis Brackwasserarten \\ Candona compressa \\ Candona sp. juv. \\ Cypridopsis aculeata \\ Cytheromorpha fuscata \\ Cytherura gibba \\ Darwinula stevensoni \\ Elofsonia baltica \\ Ilyocypris gibba \\ Limnocythere inopinata \\ Loxoconcha elliptica}

2. Seicht-marine Arten

Aurila convexa

Cythere lutea

Cytheropteron crassipinnatum

Cytheropteron latissimum

Cytheropteron nodosum

Hemicythere villosa

Hemicytherura cellulosa

Hemicytherura clathrata

Heterocythereis albomaculata 


Hirschmannia tamarindus
Hirschmannia viridis
Leptocythere crispata
Leptocythere cf. pellucida
Loxoconcha rhomboidea
Microcytherura fulva
Neocytherideis subulata
Nereina angulata
Paradoxostoma variabile
Pontocythere elongata
( $)$
Sclerochilus contortus
Semicytherura acuticostata
Semicytherura angulata
Semicytherura nigrescens
Semicytherura striata
Semicytherura undata
Urocythereis oblonga
Xestoleberis aurantia
Xestoleberis depressa

Die einmalige Probenentnahme aus diesem Gebiet kann vielleicht z.T. erklären, daß nur leere Schalen einer Art zu einem bestimmten Zeitabschnitt angetroffen werden. Trotzdem scheint es uns sehr unwahrscheinlich, daß die Anwesenheit der in den Proben so häufig vorkommenden und verschiedenartigen marinen Thanatocoenosen auf diese Weise richtig erklärt werden kann. Viel wahrscheinlicher ist es, daß diese, obgleich euryhaline Arten, nicht im Estuarium leben, sondern vom offenen Meer als Teil des Sedimentes landeinwärts, d.h. in das eigentliche Estuarium hineinverfrachtet worden sind. Dies würde übrigens gut mit dem Charakter eines Estuarium übereinstimmen: Transport landeinwärts von toten marinen Organismen und wenig oder überhaupt kein Transport meerwärts von kontinentalen Arten. Die Formen der oben genannten Süß- und Brackwasserostracoden sind nämlich sehr selten.

Als Kuriosität möchten wir erwähnen, daß wir in Probe 382 ein Exemplar der Gattung Oligocythereis gefunden haben, welche beschränkt ist auf MittelJura von N.W. Europa. Ubrigens sind in diesem Gebiet keine prequartären Schalen angetroffen worden.

\section{[II. DIE OSTRACODEN BIOCOENOSEN UND THANATOCOENOSEN IM ESTUARIUM}

\section{A. Die Ems}

In diesem supralitoralen, siiß- bis oligohalinen Teil des Estuarium wurden leider nicht die erwarteten Süß- oder Brackwasserfaunen angetroffen. Weil die Proben nicht direkt für Ostracoden genommen wurden (siehe Einleitung S. 81), besteht die Möglichkeit, daß man dafür nicht die günstigen Lokalitäten gewählt hat. Demgegenüber steht aber, daß die Biologin Frau A. P. C. DE Vos bei dieser Probenentnahme in der Ems teilgenommen und bestimmt darauf geachtet hat, daß nicht nur Lokalitäten gewählt wurden, wo überhaupt keine lebenden Ostracoden erwartet werden konnten. Wie dem auch sei, die Proben aus der Ems enthielten weder lebende noch tote Ostracoden. Merkwürdigerweise wurden aber in Probe 214 einige lebende Exemplare der Arten Cyprideis torosa, Leptocythere lacertosa und juvenile Leptocytheren gefunden. Auch ist in dieser Probe eine marine Thanatocoenosis vorhanden, welche viele in Teil II b. erwähnte Arten enthält. Dieses totale Faunenbild ist typisch für die eulitorale, mesohaline Dollart und die in 214 gefundenen Arten können auch nicht als typisch für die eigentliche Emsfauna angesehen werden.

*) Es ist nun außer Zweifel, daB der Gattungsname Hemicytherideis ein Synonym von Pontocythere ist (Schrifttum 4, S. 45, „Remarques ${ }^{\text {(4). }}$.
Es ist übrigens sehr zu bedauern, daß dieser supralitorale süß- bis oligohaline Teil des Estuariums keine Ostracoden enthielt, weil der Übergang von Süßbis Schwachbrackwasserarten nach der mesohalinen Dollart-fauna, ökologisch gesehen, von großem Interesse gewesen wäre.

\section{B. Der Dollart}

Von diesem eulitoralen, sublitoralen und mesohalinen Teil des Estuariums wurde eine große Anzahl von Proben untersucht. Aus den Daten des K.N.M.I. ersieht man, daß der Salzgehalt des Dollarts etwas wechseln kann, z.B. im Jahre 1956 war er niedrig $( \pm 10 \%$ ), in $1954 \pm 12 \%$. Detaillierte Bestimmungen des Salzgehaltes zur Zeit der Probenentnahme fehlen, doch es ist sicher, daß der Dollart ein echter mesohaliner Brackwasserbereich ist. Die Proben aus der Dollart sind z.T. profilweise genommen (s. Úbersichtskarte, Profil I, II, III, IV). Diese Profile lieferten uns eine mäßig reiche bis sehr individuenreiche lebende Ostracoden-fauna.

\section{Profil I (Fig. 1)}

Von diesem Profil wurden folgende Proben untersucht: $39,43,48,52,57$. Der Boden besteht hauptsächlich aus Lehm. (Für die detaillierten Dater über die Lithologie dieser Proben siehe den Beitrag von 
Wiggers). Wie Fig. 1 zeigt, enthalten die Proben eine reiche bis sehr individuenreiche Biocoenosis mit den folgenden Arten:

\section{Cyprideis torosa}

Leptocythere castanea

Leptocythere lacertosa (nur 1 Exemplar)

Leptocythere spp. juv.

„Thanatocoenosis I" enthält nur leere Schalen der oben erwähnten Arten. In den Proben 39 und 43 ist außerdem "Thanatocoenosis II" vorhanden. Sie besteht aus Schalen von seicht marinen Arten, welche im Dollart nicht lebend vorkommen (s. Teil II, S. 83). Diese Thanatocoenosis fehlt in den dicht bei der Küste gelegenen Proben. Das Gesamtprofil liegt im Eulitoral.

\section{Profil II (Fig. 2)}

Untersucht wurden die folgenden Proben: 128, 131, $133,135,137,139,141,143,145,147,149,154$, 158, 163. Der Boden besteht vorwiegend aus Sand. Die Biocoenosis der untersuchten Proben ist recht reich: Jugendexemplare der Gattung Leptocythere dominieren, weniger zahlreich tritt Leptocythere lacertosa auf, während Leptocythere castanea selten ist. Cyprideis torosa fehlt völlig in der lebenden Fauna, obwohl sie in Thanatocoenosis I vorhanden ist. Die tote marine Fauna (Thanatocoenosis II) ist in den meisten Proben vorhanden, weiter kommen sehr selten einige leere Schalen von Süß- bis Brackwasserostracoden vor, welche wir vereinigt haben zur "Thanatocoenosis III“.

Auch dieses Profil liegt ganz im Eulitoral.

Profil III (Fig. 3)

Von diesem Profil haben wir folgende Proben untersucht: $165,167,170,172,174,176,178,180,181$, $183,185,187,189,191,193,194,195,197,201$, 205, 206 und 211.

Der Boden besteht an der Küste aus Lehm und sandigem Lehm, weiter meerwärts wird er sandig. Aus Fig. 3 ersieht man, daß in den an der Küste genommenen Proben (211, 206, 205, 201, 197, 195, 194) die Ostracodenfauna quantitativ individuenreich ist u.zw. mit den folgenden Arten:
Cyprideis torosa

Leptocythere castanea

Leptocythere spp. juv.

In den weiter meerwärts genommenen Proben findet man nur juvenile Leptocytheren, vergesellschaftet mit einigen Exemplaren der Art Leptocythere lacertosa.

Auffallend ist, daß in den küstennahen Proben die marine Thanatocoenosis (II) fehlt, während sie in den anderen Proben immer durch einige oder mehrere Arten vertreten ist (s. auch Profil I, Fig. 1). In den Proben unseres Profiles III kommen auch wieder einige leere Schalen von Süß- bis Brackwasserostracoden vor (Thanatocoenosis III).

Von diesem Profil liegen die Proben 165 und 181 im Sublitoral, die übrigen im Eulitoral.

Profil IV (Fig. 4)

Untersucht wurden die Proben 222, 228, 233, 234, 236, 238, 240. Der Boden besteht hauptsächlich aus sandigem Lehm. Die Biocoenosis dieser Proben ist recht individuenreich, Jugendformen von Leptocythere dominieren auch hier wieder.

Weiter haben wir noch folgende Arten angetroffen:

Cyprideis torosa

Leptocythere castanea

Leptocythere lacertosa

Die marine Thanatocoenosis (II) ist in allen Proben vorhanden. Auch kommen in diesem Profil wieder einige leere Schalen von Süß-bis Brackwasserarten vor.

Das volle Profil liegt im Eulitoral.

Außer den profilveise genommenen Proben sind auch noch eine große Zahl anderer Proben im Dollart genommen, vorwiegend in den Prielen, d.h. im Sublitoral. Im allgemeinen war die lebende Fauna hier recht individuenarm, was wohl im Zusammenhang mit der Strömung in diesen Prielen stehen wird. Dies gilt vor allem für die größeren Priele. Betrachten wir zunächst die Proben aus dem Schankersdiep, Grote Gat und der Mündung des Dollarts. Hiervon wurden untersucht: $7,8,10,11,12,13,14,15,16,17,18$, $19,20,22,23,25$ und 27 (s. Ubersichtskarte EmsEstuarium). Die Ergebnisse folgen in Tabellenform:

\begin{tabular}{|c|l|l|l|}
\hline Probe Nr. & Lithologie & \multicolumn{1}{|c|}{ Bincoenosis } & \multicolumn{1}{c|}{ Thanatocoenosis } \\
\hline 7 & Ton & Einige juvenile Leptocytheren & $\begin{array}{c}\text { Eine leere Klappe einer juvenilen } \\
\text { Leptocythere } \\
\text { Einige leere Klappen von Cyprideis } \\
\text { torosa }\end{array}$ \\
$\begin{array}{c}\text { (nicht im Priel, } \\
\text { sondern auf der } \\
\begin{array}{c}\text { Oost-Friesche } \\
\text { Platte im Eulitoral) }\end{array}\end{array}$ & toniger Lehm & $\begin{array}{c}\text { Sehr viele Cyprideis torosa, einige } \\
\text { juvenile Leptocytheren }\end{array}$ \\
\hline
\end{tabular}




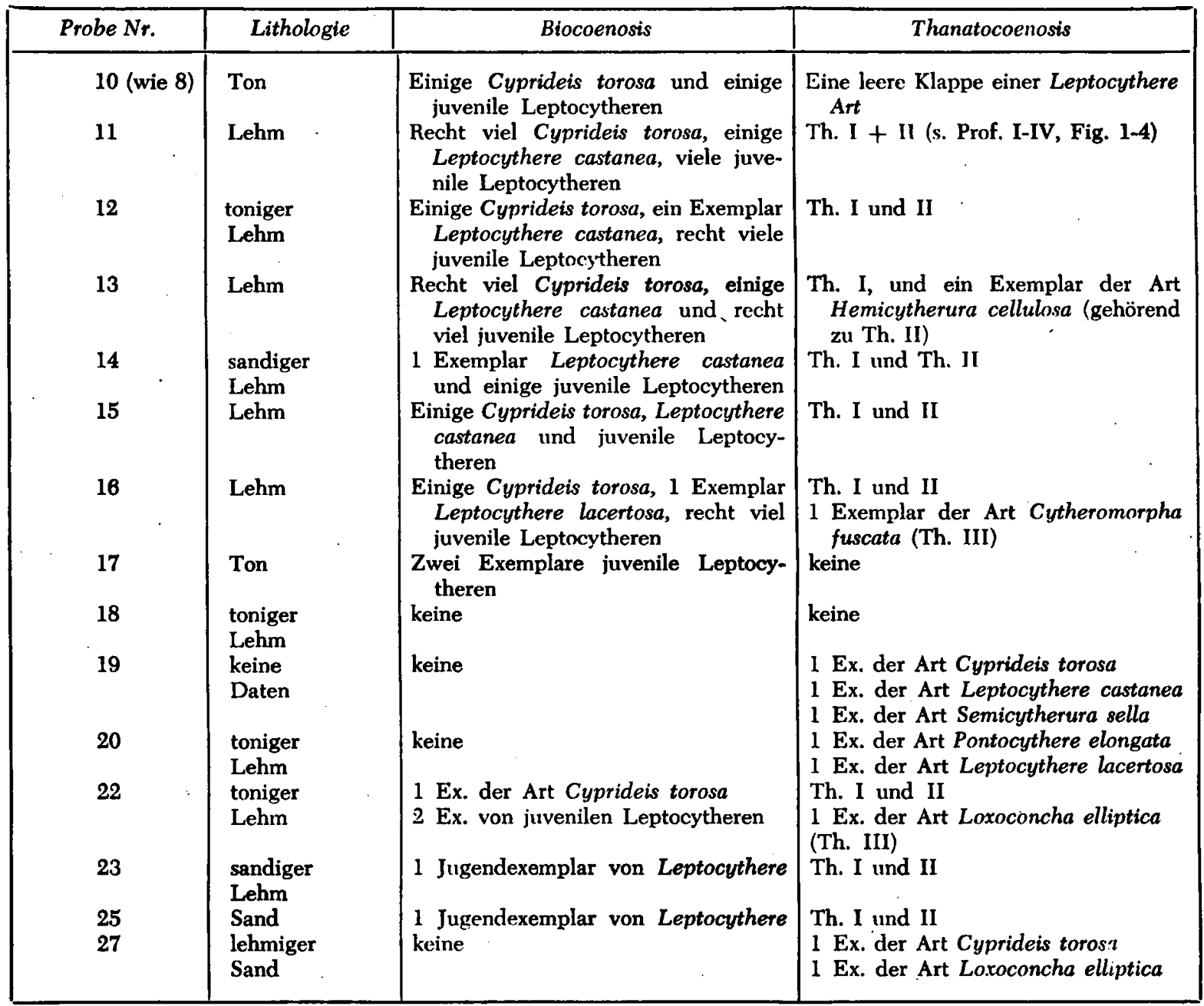

Weiter wurden noch einige Proben aus den Prielen zwischen Hering- und Reider Plate untersucht (Nr. $60,67,68,71)$.

Die Ergebnisse sind wie folgt:
1 lebende juvenile Leptocythere angetroffen. Th. I und II sind gut vertreten, während auch eine Schale von einer juvenilen Candona gefunden wurde (Th. III).

\begin{tabular}{|c|c|c|c|}
\hline Probe $\mathrm{Nr}$. & Lithologie & Biocoenosis & Thanatocoenosis \\
\hline $\begin{array}{l}60 \\
67 \\
68\end{array}$ & $\begin{array}{l}\text { sandiger } \\
\text { Lehm } \\
\text { Sand } \\
\text { Sand } \\
\text { toniger } \\
\text { Lehm }\end{array}$ & $\begin{array}{l}\text { Einige Cyprideis torosa, viele juvenile } \\
\text { Leptocytheren } \\
1 \text { Ex. der Art Leptocythere lacertosa } \\
\text { I Jugendform von Leptocythere } \\
\text { keine }\end{array}$ & $\begin{array}{l}\text { Th. I, II und III } \\
\text { Th. I, II und III } \\
\text { Th. I und II } \\
1 \text { Ex. der Art Loxoconcha elliptica } \\
\text { (Th. III) } \\
\text { Th. I } \\
1 \text { Ex. der Art Microcytherura fulva } \\
\text { (Th. II) }\end{array}$ \\
\hline
\end{tabular}

Schließlich wurden noch die Proben 221 und 241 aus der Mündung der Ems untersucht. In Probe 221 wurden keine Ostracoden gefunden. In 241 wurde
C. Das Gebiet zwischen Dollart und Rottumeroog In diesem eulitoralen, sublitoralen und polyhalin bis marinen Teil des Estuarium wurden auch viele Pro- 
ben genommen, welche wir z.T. untersucht haben. Aus der Isohalinenkarte des K.N.M.I. ersieht man, daB im Jahre 1954 im Gebiet zwischen Dollart und „Hond“ ( \pm Höhe der Probe 314) das Wasser polyhalin war. Da die von uns untersuchten Proben aus diesem Gebiet auch 1954 genommen wurden, können wir also sicher sein, daß unsere Faunen tatsächlich aus polyhalinem Milieu stammen.

Wo genau die Grenze zwischen polyhalinem und marinem Wasser liegt, ist nicht bekannt, $d a$ in dem Gebiet zwischen Probe 314 und Rottumeroog keine Salzgehaltbestimmungen gemacht wurden. Es ist aber wahrscheinlich, daß die Profile VI, VIII und IX schon in mehr oder weniger marinem Wasser liegen.

Die Proben sind, wie das auch im Dollart z.T. der Fall war, profilweise genommen (s. Ubersichtskarte, Profil V, VI, VII, VIII, IX).

Einige Profile enthielten eine individuenreiche, andere dagegen eine sehr individuenarme, lebende Fauna. Wir besprechen zunächst die Profile $\mathrm{V}$ und VII, welche im echten polyhalinem Gebiet liegen.

\section{Profil V (Fig. 5)}

Nur einige Proben aus diesem Profil wurden untersucht, u.zw. 263, 268, 270, 274, 278, 285, 291, 294. 298, 301.

Der Boden besteht hauptsächlich aus Sand (s. Beitrag von Wiggers).

Aus Fig. 5 ersieht man, da $B$ die lebende Ostracoden-fauna mäßig individuenreich ist. Leptocythere lacertosa und juvenile Leptocytheren kommen regelmäßig vor, in Probe 294 ist diese Leptocythere-fauna sogar sehr reich. Weiterhin kommen in einigen Proben sehr selten lebende Exemplare von Elofsonia pusilla und Semicytherura sella vor, welche im Dollart nicht lebend angetroffen wurden. "Thanatocoenosis I" enthält die leeren Schalen der oben erwähnten Arten. Die seicht-marinen Arten, die auch in diesem Gebiet nicht lebend vorkommen, sind wieder unter "Thanatocoenosis II" und die auch wieder sehr seltenen leeren Schalen von Süß- bis Brackwasserarten unter „Thanatocoenosis III“ erwähnt.

Das ganze Profil liegt im Eulitoral.

\section{Profil VII}

Die Proben aus diesem Profil sind so arm an Ostracoden (sowohl lebende als auch tote), daß kein Diagramm gezeichnet wurde. Der Boden besteht hauptsächlich aus sandigem Lehm. Nur einige Exemplare von Leptocythere lacertosa und juvenilen Leptocytheren wurden gefunden. In einigen Proben kommen außerdem noch einige Schalen von Arten vor, die zu Thanatocoenosis II gehören.

Das Profil liegt im Eulitoral.

\section{Profil VI}

Dieses Profil liegt in vermutlich rein marinem Wasser. Der Boden besteht aus Sand und lehmigen Sand. Auch dieses Profil ist äußerst arm an Ostracoden, soda $B$ auch kein Diagramm gezeichnet wurde. Die Fauna stimmt mit der in Profil VII erwähnten überein.

Frau A. P. C. DE Vos fand in diesem Profil einige reichere lebende Faunen; sie erwähnt die Arten:

Leptocythere lacertosa

Paradoxostoma normani

Semicytherura sella

Das Profil liegt ganz im Eulitoral.

\section{Profil VIII (Fig. 6)}

Von diesem Profil wurden die Proben 355, 358, 362, $365,369,372,376$ und 378 untersucht. Der Boden besteht vollständig aus Sand. Die lebende Ostracodenfauna ist sehr arm: einige Exemplare von Leptocythere lacertosa, einige juvenile Leptocytheren und eine einzige Semicytherura sella. Die marine Thanatocoenosis (II) ist in einigen Proben gut vertreten, auch wurden einige leere Schalen von Süß-bis Brackwasserformen angetroffen (Thanatocoenosis III).

Das Profil liegt im Eulitoral und das Wasser ist vermutlich vollkommen marin.

\section{Profil IX (Fig. 7)}

Die untersuchten Proben (379, 382, 387, 390, 391, $393,395,401)$ aus diesem marinen eulitoralen Profil enthielten eine sehr individuenreiche lebende Ostracodenfauna, wie aus Fig. 7 deutlich ersichtlich ist. Leptocythere lacertosa und juvenile Leptocytheren kommen sehr oft vor, auch Elofsonia pusilla und Semicytherura sella sind, weniger zahlreich, vorhanden.

Auffallend ist, da $\beta$ in den küstennahen Proben (401, 395, 393) die marine Thanatocoenosis (II) fehlt. In den übrigen Proben ist sie durch einige oder mehrere Arten vertreten (s. auch Profil I, Fig. 1 und Profil III, Fig. 3).

Der Boden von Profil IX besteht ganz aus Sand.

In diesem Gebiet wurden auch noch viele verstreut liegende Proben genommen. Die Proben 305, 306, 307 und 308 liegen aber noch mehr oder weniger profilweise dicht an der Küste westlich von der Hond Plate. Der Boden besteht hauptsächlich aus Lehm. 
Die lebende Fauna scheint sehr individuenarm zu sein; die folgende Arten wurden angetroffen:

Leptocythere lacertosa

Leptocythere sp. juv.

Semicytherura sella

Elofsonia pusilla

Cytherois fischeri

In den Proben kommen auch noch einige leere Schalen vor, die zu Thanatocoenosis II gehören.
Die übrigen Proben wurden in den großen Prielen genommen, und es zeigte sich, daß die lebende Fauna entweder fehlt oder nur von einem einzelnen Exemplar vertreten ist. Die leeren Schalen der marinen Thanatocoenosis kommen aber in diesen Proben recht zahlreich vor.

Die Ergebnisse dieser verstreuten Proben sind folgende:

1. Mündung der Ems und Oost Friesche Gaatje

\begin{tabular}{|c|l|l|l|}
\hline Probe Nr. & \multicolumn{1}{|c|}{ Lithologie } & \multicolumn{1}{|c|}{ Biocoenosis } & \multicolumn{1}{c|}{ Thanatocoenosis } \\
\hline 242 & $\begin{array}{l}\text { keine Daten } \\
\text { Sand }\end{array}$ & $\begin{array}{l}\text { 1 Ex. der Art Cyprideis torosa } \\
\text { keine }\end{array}$ & $\begin{array}{l}\text { Th. I, II und III } \\
\text { Viele leere Klappen von Cyprideis } \\
\text { torosa. Zwei leere Klappen Ponto- } \\
\text { cythere elongata } \\
\text { Th. I, II und III } \\
\text { keine } \\
\text { keine } \\
\text { Einige leere Klappen von Pontocy- } \\
\text { there elongata (Th. II) }\end{array}$ \\
303 & $\begin{array}{l}\text { keine Daten } \\
\text { keine Daten } \\
\text { keine Daten } \\
\text { sandiger Lehm }\end{array}$ & $\begin{array}{l}\text { 1 Jugendform von Leptocythere } \\
\text { keine } \\
\text { keine } \\
\text { keine }\end{array}$ &
\end{tabular}

2. „Bocht van Watum“

\begin{tabular}{|c|c|c|c|}
\hline Probe Nr. & Lithologie & Biocoenosis & Thanatocoenosis \\
\hline $\begin{array}{r}30 \\
33 \\
34 \\
35 \\
36 \\
37 \\
312 \\
311\end{array}$ & $\begin{array}{l}\text { sandiger Lehm } \\
\text { keine Daten } \\
\text { Sand } \\
\text { toniger Lehm } \\
\text { Sand } \\
\text { Sand } \\
\text { keine Daten } \\
\text { keine Daten }\end{array}$ & $\begin{array}{l}\text { keine } \\
\text { keine } \\
\text { keine } \\
\text { keine } \\
\text { keine } \\
1 \text { juvenile Lcptocythere } \\
\text { keine } \\
1 \text { Ex. der Art Paradoxostoma normani }\end{array}$ & $\begin{array}{l}\text { keine } \\
\text { keine } \\
\text { Th. I und II } \\
\text { keine } \\
\text { Th. I, II und III } \\
\text { Th. I, II und III } \\
\text { Th. I und II } \\
\text { Einige leere Klappen von Cyprideis } \\
\text { torosa und eine juvenile Leptocy- } \\
\text { there }\end{array}$ \\
\hline
\end{tabular}

\section{Oude Wester Eems}

\begin{tabular}{|c|c|c|c|}
\hline Probe $\mathrm{Nr}$. & Lithologie & Biocoenosis & Thanatocoenosis \\
\hline $\left.\begin{array}{l}317 \\
318 \\
319\end{array}\right\}$ & keine Daten & $\begin{array}{l}\text { keine } \\
\text { keine } \\
\text { keine }\end{array}$ & $\begin{array}{l}\text { keine } \\
\text { Eine leere Klappe von Cyprideis to- } \\
\text { rosa, einige Pontocythere elongata } \\
\text { Zwei leere Klappen von Cyprideis } \\
\text { torosa und drei von Pontocythere } \\
\text { elongata }\end{array}$ \\
\hline
\end{tabular}

Probe 329, genommen an der Südwestküste von Rottumeroog enthielt auch keine lebende Fauna. In dieser Probe wurden nur einige leere Klappen von Cytherois fischeri, Pontocythere elongata, Leptocythere cf. pellucida und einige juvenile Leptocytheren gefunden. 


\section{ABSCHLIESSENDE FOLGERUNGEN}

Aus den in Teil III gegebenen Daten ersieht man, $\mathrm{da} \beta$ die lebende Ostracodenfauna, die in den untersuchten Proben des Ems-Estuarium angetroffen wurde, sehr artenarm ist. In dem supralitoralen, süß- bis oligohalinen Teil des Estuarium (der eigentlichen Ems) wurden überhaupt keine Ostracoden gefunden.

In dem eulitoralen, mesohalinen Teil des Dollarts waren folgende Arten lebend vorhanden "):

Cyprideis torosa

Leptocythere castanea

Leptocythere lacertosa

Es stellte sich heraus, daß die beiden erstgenannten Arten in recht großer Anzahl gemeinsam in den Küstentonen vorkommen (Profil I, Profil III, Probe 8). In den sandigeren Teilen des Dollarts fehlt Cyprideis torosa fast vollkommen und ist Leptocythere castanea viel weniger reich vertreten. Beide Arten sind, nach ihren auto-ökologischen Daten (Schrifttum 4, S. 40, 54) Formen, welche besonders in Lagunengebieten und in Flußmündungen auftreten. Unsere Studien bekräftigen diese Daten, doch wir können sie auf Grund unserer Untersuchungen noch weiter klarstellen.

Synökologisch gesehen treten Cyprideis torosa und Leptocythere castanea gemeinsam auf in einem Biotop mit folgenden Merkmalen:

Boden tonig mit Pflanzenresten

Lebensraum eulitoral

Bereich mesohalin.

Wir können also sagen, daß die Küstentone des Dollarts eine charakteristische Ostracodenassoziation enthalten, wobei noch dazu kommt, daß die dort gefundene Thanatocoenosis die Biocoenosis representiert. (Nur Thanatocoenosis I; Teil III). Dies ist von großem Interesse für die Paläo-ökologie der Quartär Ostracoden: Eine Paläothanatocoenosis die die nur obengenannten Arten in großer Quantität enthält, gibt also eine gutfundierte Milieubestimmung.

Aus Teil III ersieht man weiter, daß in den sandigeren Teilen des eulitoralen Dollarts die lebende Ostracodenfauna viel weniger individuenreich ist. Hier treten die Arten Leptocythere lacertosa und Leptocythere castanea auf, beide übrigens recht selten. Vielleicht sollte noch erwähnt werden, daß lebende Ostracoden im ganzen Estuarium fast immer auf oder zwischen Pflanzenresten gefunden wurden, was eigentlich selbstverständlich ist, da diese Litoralostracoden Pflanzenfresser sind. In Sandproben ohne Pflanzenreste kommen sie dann auch nicht vor. Der

-) Juvenile Leptocytheren werden außer Acht gelassen.
Literaturhinweis Substrat: „Sand“ für einige Leptocythere-arten scheint uns deshalb fraglich. Die Biocoenosen des sandigen eulitoralen Dollarts sind zu individuenarm, um weitgehende ökologische Folgerungen zu ziehen. Im sandigen Dollart kommen weiter in fast allen Proben die bereits in Teil II und III erwähnte Thanatocoenosis von seicht marinen Arten (Th. II) vor. Diese Thanatocoenosis ist bisweilen recht artenreich. Das sagt also, daß der Quartärpaläontologe, der gezwungenermaßen nur mit leeren Schalen arbeiten muß, die tatsächliche Biocoenosis in solch einem Falle nicht mehr rekonstruieren könnte, und kaum auf ein mesohalines Wattmilieu schließen würde.

Wir haben bereits in Teil III darauf hingewiesen, daß marine Schalen als Teile des Sedimentes aus der Nordsee in das Estuarium transportiert wurden. Dies bringt uns zu dem Schluß, daß der Dollartsand sicher z.T. aus der Nordsee stammt. Dies gilt übrigens auch für das polyhalin-marine, eulitorale Gebiet zwischen Dollart und Rottumeroog. Aus all diesem ersieht man nun, wie unentbehrlich es ist, bei einem ökologischen Studium tote und lebende Exemplare zu trennen. Ein Studium, nur auf Thanatocoenosen gestützt und die daraus gezogenen Milieufolgerungen, müssen wohl zu vollkommen falschen Resultaten auf paläoökologischem Gebiet führen.

Im eulitoralen Gebiet zwischen Dollart und Rottumeroog gibt es keinen Faunenunterschied zwischen dem östlichen polyhalinen und dem westlichen mehr marinen Teil. Die lebende Ostracodenfauna ist aber von der im Dollart verschieden.

Auch im erstgenannten Gebiet treten die quantitativ individuenreichsten Faunen direkt an der Küste auf (s. Profil IX).

Die Fauna besteht aus folgender Arten:

Leptocythere lacertosa (dominierend)

Elofsonia pusilla

Semicytherura sella.

Die sehr küstennahen Proben sind auch hier wieder frei von der marinen Thanatocoenosis. Es zeigte sich, $\mathrm{da} ß$ auch in anderen Proben aus diesem Gebiet neben der dominierenden Leptocythere lacertosa, die beiden anderen Arten vorkommen, sodaß man hier von einer Assoziation sprechen kann, wobei die quantitativ armen Arten gerade charakteristisch sind. Das Biotop dieser Assoziation kann folgendermaßen definiert werden:

Boden sandig (mit Pflanzenresten)

Lebensraum eulitoral

Bereich polyhalin-marin. 
Wie schon oben erwähnt, ist auch in diesem Gebiet die marine Thanatocoenosis (Th. II) vorhanden, was eine Milieubestimmung nur auf Grund von leeren Schalen wieder sehr schwierig machen würde.

Schließlich wollen wir noch erwähnen, daß in diesem Gebiet nur ein einziges Mal auch die Arten Cytherois fischeri und Paradoxostoma normani lebend gefunden wurden.

In den sublitoralen großen Prielen des Ems-Estuarium wurden praktisch keine lebenden Arten angetroffen, wie wir das erwartet hatten, u.z. wegen der starken Gezeitenströmungen, die in diesen großen Prielen auftreten. Die marine Thanatocoenosis war in einige dieser Proben gut vertreten.

Leere Schalen von Süß- und Brackwasserarten (Ems?) wurden sehr sporadisch im ganzen Estuariumgebiet gefunden.

Die Armut von lebenden Ostracodenarten im eulitoralen, mesohalinen und polyhalin-marinen Teil des Estuarium (Wattengebiet) stimmt gut mit den Untersuchungen in analogen Gebieten überein, wie z.B. das Mangrove-Estero-Wattengebiet von El Salvador (Schrifttum 1). Auch in diesem Gebiet treten nur sehr wenige Ostracodenarten im eulitoralen Bereich auf. Ein Wattengebiet scheint also, was die Ostracoden betrifft, gekennzeichnet zu sein durch Artenarmut.

Offenbar hält das typische Wattmilieu (eine Kombination von sedimentpetrologischen, hydrologischen und biologischen Kriteria) das Eintreten fast aller euryhaliner, eurythermer und seicht mariner Arten fern (Schrifttum 4, Fig. III, S. 112). Diese Arten sind aber als leere Schalen im Estuarium vorhanden, jedoch scheinen sie uns, wie schon früher erwähnt, Teile des Nordseesedimentes zu sein.

Auch sahen wir, daß diese Thanatocoenosis nicht vorhanden ist in den an der Küste gelegenen Sedimenten.

Die Folgerung, wichtig für Studien der Paläoökologie, ist also, daß in fossilen Wattbildungen die Randsedimente das sauberste Bild der Biocoenosen geben.

Es wäre sehr interessant gewesen, wenn auch Proben aus dem Nordseegebiet nördlich von Rottumeroog und im Wattenmeer in der Richtung von Schiermonnikoog und Ameland genommen wären. Aus diesem Gebiet haben wir in unserer Publikation (1957, Schrifttum 4, Fig. XIII-XVI) die Ergebnisse von Untersuchungen an dortigen Thanatocoenosen wie folgt beschrieben (S. 120): „Les valves vides que nous avons trouvées appartiennent, en général, à des espèces qui éventuellement, pourraient vivre à l'endroit même de l'échantillonage, compte tenu des données fournies par Elofson pour le Skager-Rak. Les thanatocènoses pourraient donc représenter plus ou moins, dans ce cas, la faune vivante“.

Auf Grund der jetzigen Untersuchung des EmsEstuarium meinen wir, daß der größte Teil dieser Fauna nicht dort gelebt hat, sondern, wie im Estuarium, auch als Teil des Sedimentes von der Nordsee hier abgesetzt wurde. Sehr empfehlenswert wäre es also, aus diesem Gebiet noch mehrere Proben zu nehmen und lebende und tote Exemplare zu trennen. Um das faunistische Bild unserer Wattgebiete zu vervollständigen, rekommandieren wir gründliche Untersuchungen des oben genannten Gebietes.

\section{SCHRIFTTUM}

1) Haftmann, G. (1956) - Zur Kenntnis des MangroveEstero-Gebietes von El Salvador und seiner OstracodenFauna. Kieler Meeresforschungen. Band XII, Heft 2, S. 219-248.

la) Hartmann, G. (1957) - Neue Funde von Muschelkrebsen (Ostracoden) im Gebiet der Nordseeküste und der Kieler Bucht. Schriften des Naturwiss. Vereins für Schleswig-Holstein, Band XXVIII, Heft 2.

2) Remane, A. (1940) - Einführung in die zoologische
Ökologie der Nord- und Ostsee. Die Tierwelt der Nordund Ostsee, Lief. XXXIV, Teil la, Leipzig.

3) Välukangas, I. (1933) - Uber die Ostsee als Brackwassergebiet. Verh. Int. Verein. Limm. VI:I Stuttgart.

4) Wagner, C. W. (1957) - Sur les Ostracodes du Quaternaire récent des Pays-Bas et leur utilisation dans l'étude géologique des dépôts holocènes. Thèse Sorbonne, Paris. Mouton \& Cie, La Haye. 


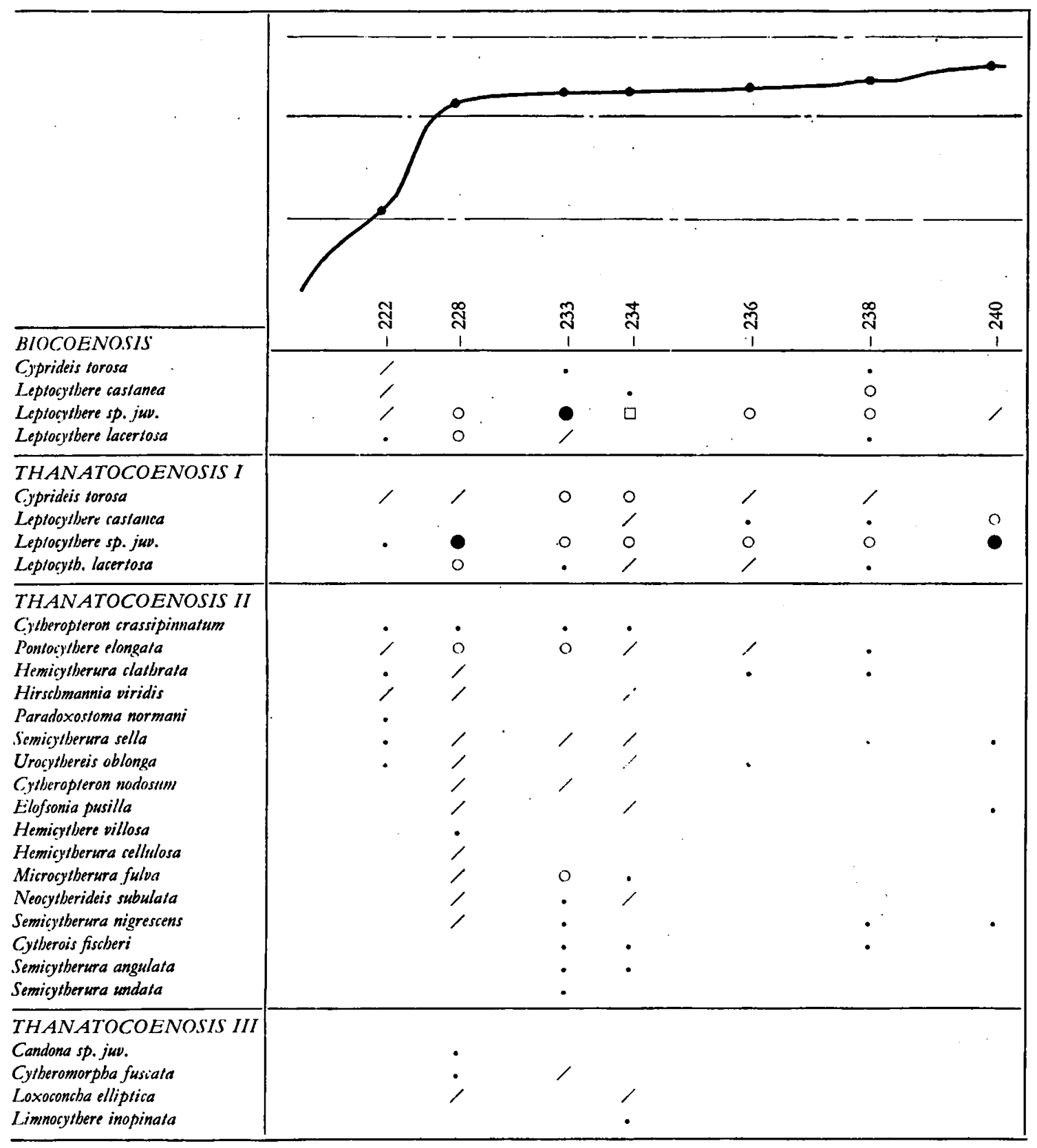

M. H.W.

N.A.P.

M. N.W'.

Fig. 4. Profil IV. Legende s. Fig. 1. 


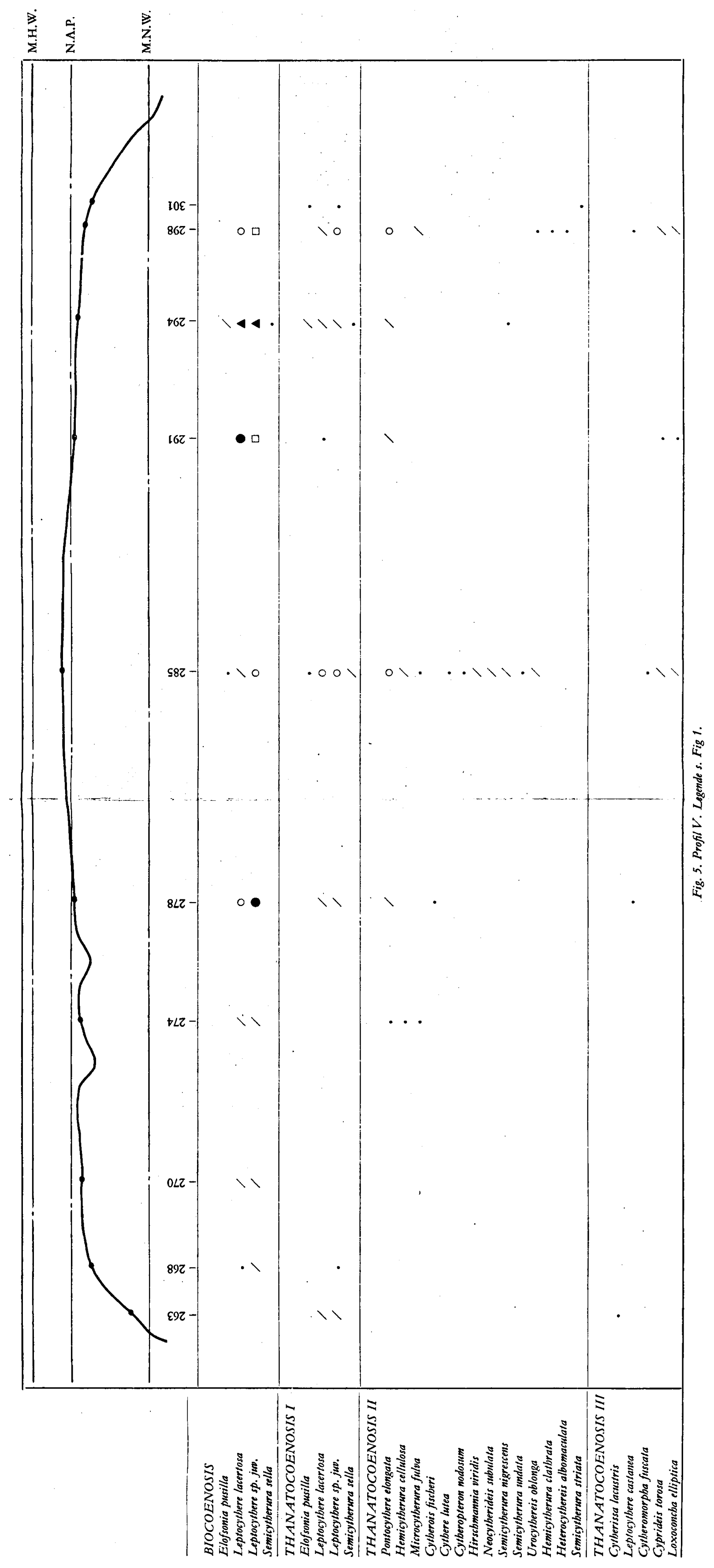




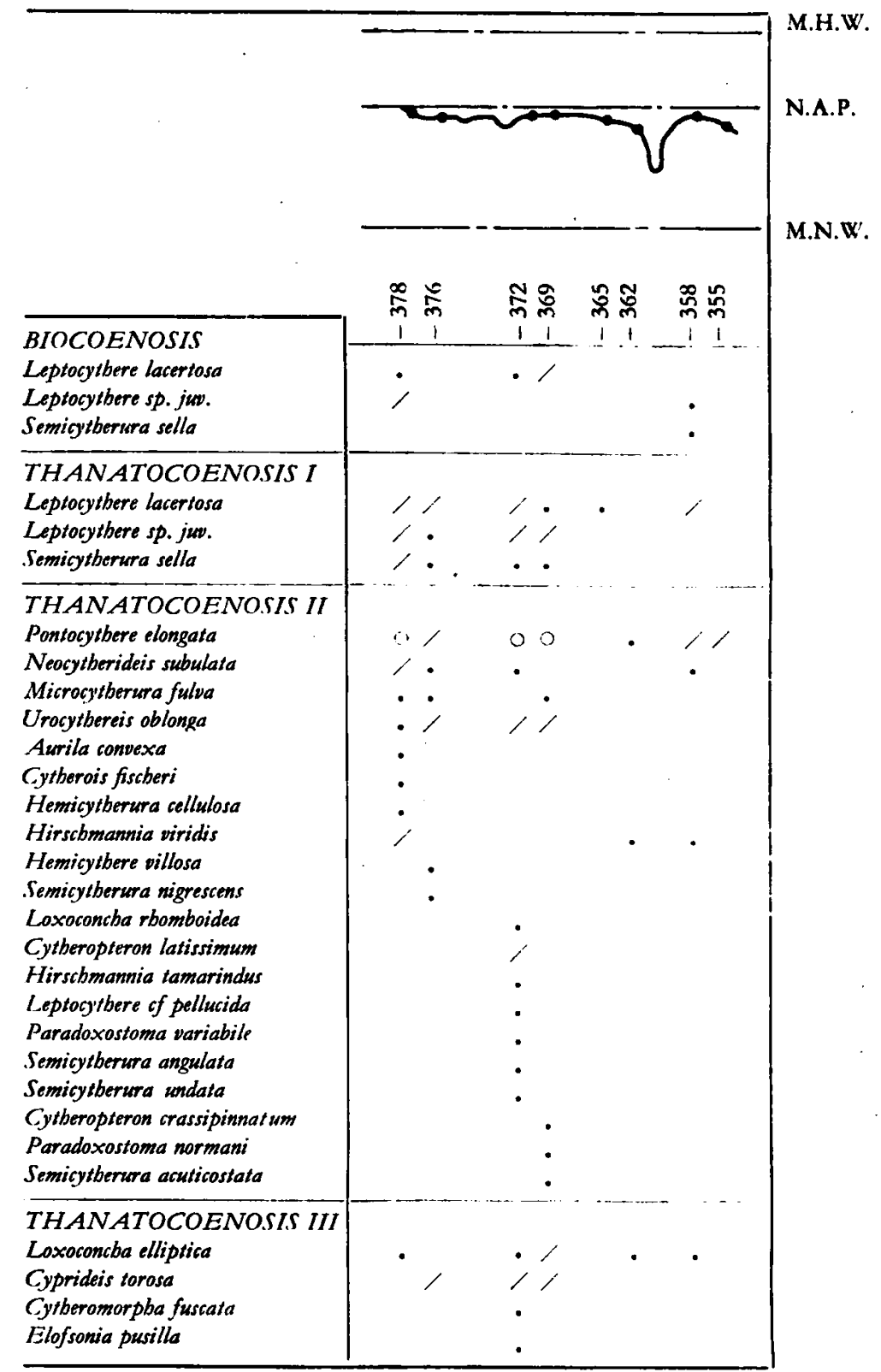

Fig. 6. Profil VIII. Legende s. Fig. 1. 
M. H.W.

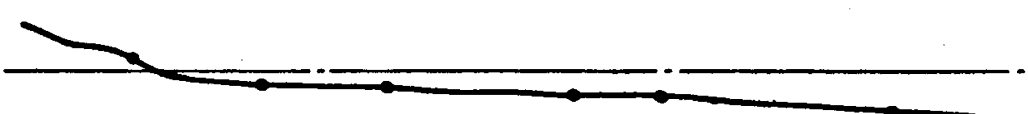

N. A.P.

BIOCOENOSIS

Elofsomia pusilla

Leptocythere lacertosa

Leptocythere sp. juw.

Semicytberura sella

THANATOCOENOSIS I

Elofsonia pusilla

Leptocythere lacertosa

Leplocythere sp. juv.

Semicytberura sella

THANATOCOENOSIS II

Neocytberideis subulata

Aurila convexa

Hemicythere villosa

Pontocythere elongata

Hirschmannia viridis

Urocythereis oblonga

Xestoleberis sp. jwo.

Microcytberura fulva

Cytbere lutea

Cytberois fiscberi

Cytheropteron latissimum

Hemicytberura cellulosa

Paradoxostoma normani

Semicytherura nigrescens

THANATOCOENOSIS III

Cyprideis torosa

Loxoconcha elliptica

Cytberomorpba fuscata

\begin{tabular}{|c|c|c|c|c|c|c|c|}
\hline $\bar{q}$ & $\stackrel{n}{n}$ & $\stackrel{m}{\tilde{m}}$ & $\overline{\mathrm{n}}$ & $\underset{9}{8}$ & 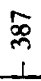 & న్లె & $\frac{2}{2}$ \\
\hline & • & & I & $\bullet$ & $\therefore$ & r & \\
\hline$\square$ & 0 & I & $\Delta$ & 0 & $\square$ & 0 & \\
\hline $\boldsymbol{\Delta}$ & 0 & $\mathbf{A}$ & $\Delta$ & $\Delta$ & $\Delta$ & $\Delta$ & 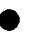 \\
\hline . & & $\Delta$ & 1 & I & $\therefore$ & , & \\
\hline
\end{tabular}

M.N.W.

$+2$

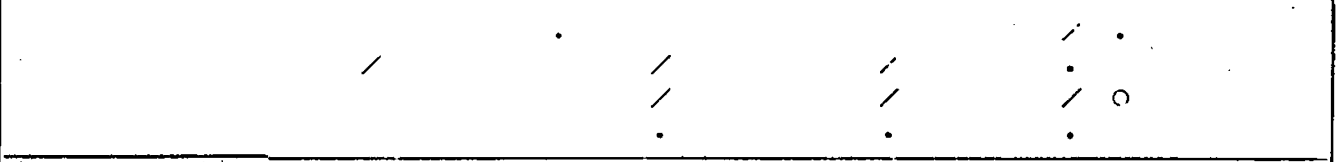

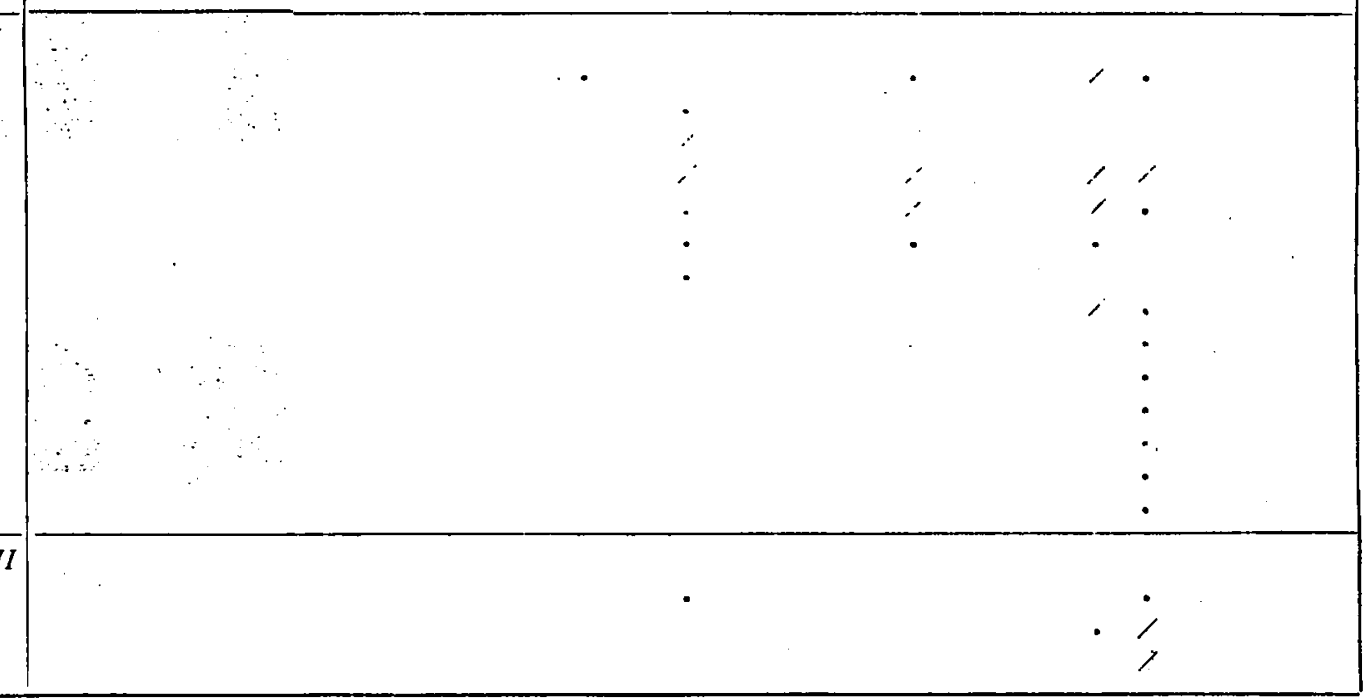

Fig. 7 Profil IX. Legende s. Fig. 1. 